\title{
GREEN SYNTHESIS AND CHARACTERIZATION OF ZnO NANOPARTICLES FROM LEAF EXTRACTS OF BARRINTONIA ACUTANGULA AND ITS ANTIBACTERIAL ACTIVITY
}

\author{
S. Renjusha ${ }^{\varpi}$ and P.H. Vaisakh \\ Department of Chemistry, N. S. S. College, Pandalam-689501(Kerala)India \\ ${ }^{\square}$ Corresponding Author: renjushasathish@gmail.com
}

\begin{abstract}
Plant-mediated synthesis of metal oxide nanoparticles is a promising alternative to the traditional physical and chemical synthesis method. Using the reduction and capping capacity of aqueous extracts from Barrintonia Acutangula leaves, biosynthesis of zinc oxide nanoparticles (ZnO-NPs) has been achieved. Using powder X-ray diffraction, ultraviolet-visible spectroscopy, scanning electron microscopy and Fourier transform infrared spectroscopy techniques, the bioreduced ZnO NPs are characterized. The development of spherical structures with an average grain size between 5 and $20 \mathrm{~nm}$ in diameter was confirmed by XRD research. The existence of stretching vibrations of-O-H, C-H C-N, C = O groups involved in the reduction and stabilization of nanoparticles was shown by FTIR spectra. The existence of spongy, spherical, porous agglomerated nanoparticles is recognized by S.E.M. images. UV-visible ZnO-NP spectra showed a high absorption peak at 370, 376, and $373 \mathrm{~nm}$. The antibacterial activity of the $\mathrm{ZnO}$ nanoparticleswas evaluated by testing against Gram-negative and Gram-positive bacteria. Synthesized $\mathrm{ZnO}$ nanoparticles' antibacterial activity is more effective.
\end{abstract}

Keywords: Antibacterial Activity, Barrintonia Acutangula Leaves, Green Synthesis, ZnO Nanoparticles, S.E.M., UV-Vis Spectrophotometer, XRD.

RASĀYAN J. Chem., Vol. 14, No.3, 2021

\section{INTRODUCTION}

Nanotechnology is expected to be the basis of many biotechnological innovations in the $21^{\text {st }}$ century and is regarded as the upcoming industrial revolution. Over the past few decades, nanomaterials have been called 'a wonder of modern medicine'. ${ }^{1}$ Owing to their superior physicochemical and biological properties during their mass phases, nanomaterials are of great significance. The development and reaction of nanoparticles and their compounds are linked to nanochemistry or nanotechnology. The nanoparticle is prepared to obtain a unique characteristic for the specific purpose of the application. ${ }^{2}$ A rapid stride in synthesis and applications of nanomaterials, in recent years, has been perceived in almost every domain of life, including health care, cosmetics, biomedical, food and feed, drug-gene delivery, environment, electronics, mechanics, catalysis, energy science, optics, chemical and space industries. ${ }^{3}$ Noble metal nanoparticles (NPs) such as gold, silver, platinum and zinc are commonly used in medical and pharmaceutical applications and a wide variety of products for the consumer trade. ${ }^{4}$ However, among the nanoparticles, zinc oxide nanoparticles play an excellent role in the biological and drug industry. ${ }^{5}$ The science we use to produce nano materials with unique sizes, shapes, and surface properties using synthetic chemistry and material chemistry. Cross-disciplinary nano science research involving physics, chemists and engineers are concerned about the need for developing eco-friendly and sustainable methods for the synthesis of nanomaterials. ${ }^{6}$ There are many methods for the synthesis of nano particles, but one of the most conventional methods is green synthesis because it is an eco-friendly, non-toxic, significantly less expensive and very pure method. Nano particles are having unique properties arising from their nano scale dimensions. Nanoparticles have many important properties and various applications in many areas such as drug, food, nutrition, electronics etc. ZnO-NPs are commercially available in the form of powders 
or dispersions, which are extensively used in calamine lotion, baby powders, ceramics, U.V. filters, ointments, paints, food additives, etc.

$\mathrm{ZnO}$ NPs have also been reported to possess potential human health benefits such as antimicrobial, ${ }^{7}$ antioxidant, ${ }^{8}$ anticorrosive ${ }^{9}$ and anticancer activity. Zinc oxide $(\mathrm{ZnO})$ is gaining attention by researchers in the recent past owing to its unique properties and abundant applications. Based on the previous literature reports, $\mathrm{ZnO}$ nanoparticles have been synthesized from various plant extracts such as Azadirachta indica, Passiflora caerulea, Aloe vera, Vitex trifolia, Trifolium pratense, Bauhinia tomentosa, Cinnamomum verum, Camellia sinensis, Artocarpus gomezianus, Durantaerecta, Moringa oleifera, Matricaria chamomilla, Olea europaea, and Lycopersicon esculentum and their antimicrobial activities were also reported. ${ }^{10}$ This has prompted us to use Barrintonia Acutangula plant extract to synthesize zinc oxide nanoparticles ( $\mathrm{ZnO} \mathrm{NPs}$ ).

Barrintonia Acutangula is the traditional medicinal plant as popularly used. It is known as the Indian Oak tree comes under the Lecythidaceae family, which is used in India by tribal people for the treatment of liver disorders, diarrheal diseases, eye diseases, worm infections and jaundice. All the parts of Barrintonia Acutangula like leaf, fruit, seed, bark, and root were used to cure and treat many hemolytic diseases, abdominal colic, malaria, diabetes, etc.

Lately, environmentally friendly single-step protocols using plant extracts without involving any extrinsic surfactants, capping agents, and/ or templates have been explored for metal NPssynthesis. ${ }^{11}$ This intrinsically green approach involves the biomolecules such as proteins, amino acids, enzymes, vitamins, alkaloids, phenolics, saponins, tannins, and terpenoids, present in plant extracts, for reduction and stabilization of metal ions. ${ }^{12}$ Some of these biomolecules act as electron shuttles in metal reduction. At the same time, other constituents are responsible for capping of resulting N.P.s, which not only controls the aggregation of NPs but also results in post-surface modification of NPs. ${ }^{13}$

Therefore, this work aimed to explore the application of Barrintonia Acutangula leaves extract as a capping and reducing agent for the synthesis of $\mathrm{ZnO}$ NPs and its various traditional applications and evaluate the antibacterial activities of the synthesized $\mathrm{ZnO}$ NPs against pathogenic organisms. Furthermore, this work opens up a door for further explorations on many plants for nanomaterial synthesis, which could be used for various applications.

\section{EXPERIMENTAL}

Zinc nitrate $\left(\mathrm{Zn}\left(\mathrm{NO}_{3}\right)_{2} \cdot 6 \mathrm{H}_{2} \mathrm{O}\right)$ was purchased from Merck Chemicals Ltd, India and was used as received without further purification. All glassware was washed with sterile distilled water and dried in an oven before use. The procedure for the synthesis was referenced from the literature.

\section{Preparation of the Leaf Extract}

Fresh leaves were collected from Barrintonia Acutangula plants in Pathanamthitta district in kerala. The leaves were washed several times with water to remove the dust particles and then sun dried to remove the residual moisture. The extract was prepared by placing $50 \mathrm{~g}$ of washed dried fine cut leaves in $250 \mathrm{~mL}$ glass beaker along with $100 \mathrm{ml}$ of sterile distilled water. The mixture was then boiled for 60 minutes until the color of the aqueous solution changes from watery to light yellow. The extract was cooled to room temperature and filtered. The extract was stored in a refrigerator in order to be used for further experiments.

\section{Preparation of Zinc oxide Nanoparticles}

For the synthesis of nanoparticles $50 \mathrm{ml}$ of Barrintonia Acutangula leaves extract was taken and boiled to 60-80 degrees Celsius using a stirrer-heater. 5 grams of Zinc Nitrate was added to the solution as the temperatures reached 60 degrees Celsius. This mixture is then boiled until it is reduced to a deep yellow colored paste. This paste was then collected in a ceramic crucible and heated in an air-heated furnace at 400 degrees Celsius for 2 hours. A light-yellow colored powder was obtained and this was carefully collected and packed for characterization purposes. The material was mashed in a mortar pestle to get a more delicate nature for characterization. 


\section{X-Ray Diffraction (XRD) Analysis}

\section{RESULTS AND DISCUSSION}

The green synthesis of ZnO NPs was confirmed by XRD analysis. The powdered sample was used by a $\mathrm{CuK} \alpha$ - X-Ray Diffractometer to confirm the presence of $\mathrm{ZnO}$ and analyze the structure. The graph showed prominent peaks corresponding to $2 \theta$ values of $31.8^{\circ}, 34.5^{\circ}, 36.3^{\circ}, 47.5^{\circ}, 56.6^{\circ}, 62.9^{\circ}, 66.4^{\circ}, 67.9^{\circ}$ and $69.1^{\circ}$ corresponding to (100), (002), (101), (102), (110), (103), (200), (112), and (201) crystal planes, respectively, which is in agreement with the previously reported work ${ }^{14}$ in the multi-plot shown in Fig.-1. The graph's peaks are in good agreement with the literature report (JCPDS File no.5-0566). The location of the peaks was compared to literature values and the presence of zinc oxide particles was confirmed. The average size of the particles was calculated using Debye-Scherrer's formula. ${ }^{15}$ The development of spherical structures with an average grain size between 5 and $20 \mathrm{~nm}$ in diameter was confirmed by XRD research.

Where:

$$
\mathrm{D}=\mathrm{K} \lambda /(\beta \cos \theta)
$$

$\mathrm{D}$ - the crystal size $(\lambda=0.15406 \mathrm{~nm})$ for $\mathrm{CuKa}$,

$\lambda-$ the wavelength of the $\mathrm{x}$-ray radiation

K- usually taken as 0.89

$\mathrm{B}$ - the line width at a half-maximum height

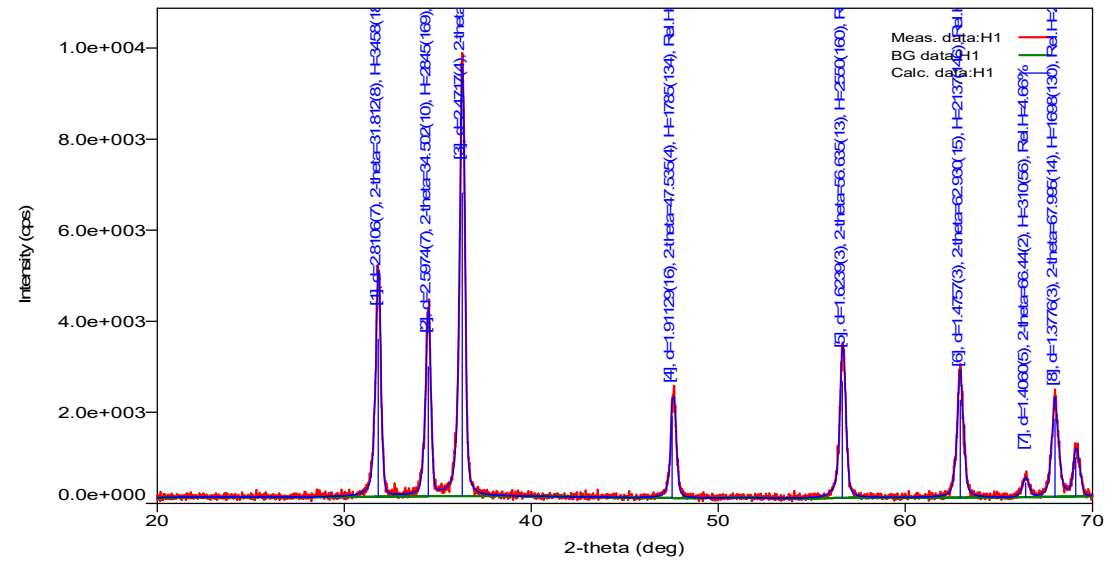

\section{Scanning Electron Microscopy(SEM)}

Fig.-1

The surface morphologies of biosynthesized $\mathrm{ZnO}$ nanoparticles were studied using S.E.M., and the results are presented in Fig.-2. The image shows spherical shapes with a diameter range of 11-25 nm. The particles are also found to be inclined together due to more capping agents that stabilize the nanoparticles. The difference in size is possibly due to the fact that the nanoparticles are being formed at different times. This aggregation/agglomeration may be caused due to polarity and electrostatic attraction of $\mathrm{ZnO}$ nanoparticles. ${ }^{16}$

\section{UV-Visible Spectral Analysis}

It was observed that sample extract showed an optical absorption band peaking at 370nm (Fig.-3). No other peak was observed in the spectrum, confirming that the synthesized product was $\mathrm{ZnO}$ only. Furthermore, it is reported that the peak positions of UV-visible spectra are related to size of nanoparticles and blue-shifted as the crystal size of the nanoparticles decreased ${ }^{17}$.Zinc oxide nanoparticles have been reported to exhibit a characteristic broad absorption peak between 330-460 nm. Thus optical absorption band peaking at $370 \mathrm{~nm}$ confirms the formation of zinc nanoparticles by Barrintonia Acutangula leaf extracts. It can be deduced from the above results that the biomolecules present in the plant extract induce the reduction of Zinc ions into zinc Oxide nanoparticles (ZnO N.P.). This reduction process is extracellular, fast and thus can be developed into easy method for nanoparticle synthesis. ${ }^{18}$ 


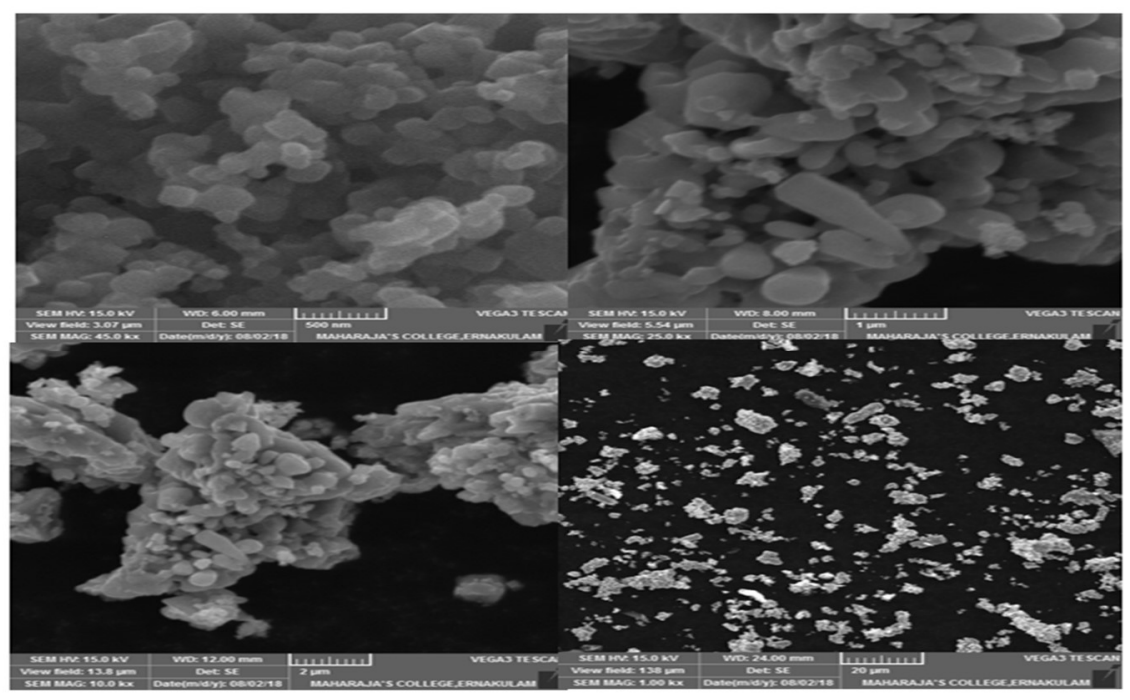

Fig.-2

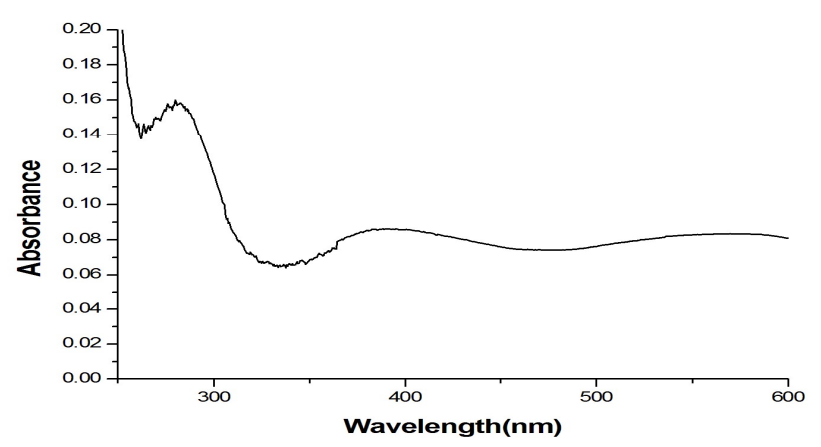

Fig. -3

\section{FTIR Spectroscopy}

The FTIR measurements are carried out to identify the possible biomolecules responsible for the production of ZnO NPs. The I.R. spectrum was taken using a resolution of $4000-400 \mathrm{~cm}^{-1}$ in the percent transmittance mode. In addition to the absorption bands of the biomolecules used as reduction and stabilization (capping) agents, the absorption peak at $850 \mathrm{~cm}^{-1}$ indicates the presence of $\mathrm{Zn}-\mathrm{O}$ stretching vibration. ${ }^{19}$ FTIR analysis of the ZnO N.P. revealed the presence of peaks at 3350, 1820, 1450, 1250, 850 $\mathrm{cm}^{-1}$. The appearance of an intense broadened band at $3350 \mathrm{~cm}^{-1}$ denotes the presence of $\mathrm{O}-\mathrm{H}$ stretching of alcohols and phenols. ${ }^{20}$ The bands at $1820 \mathrm{~cm}^{-1}$ confirm the presence of primary amines. FTIR analysis of the $\mathrm{ZnO}$ N.P. reveals the appearance of bonds that confirm the presence of certain enzymes responsible for the reduction, capping, and stability of the nanoparticles. It can be stated that the nanoparticles are shielded by the enzymes and the proteins present in the extract to help prevent the formation of clusters (Fig.-4).

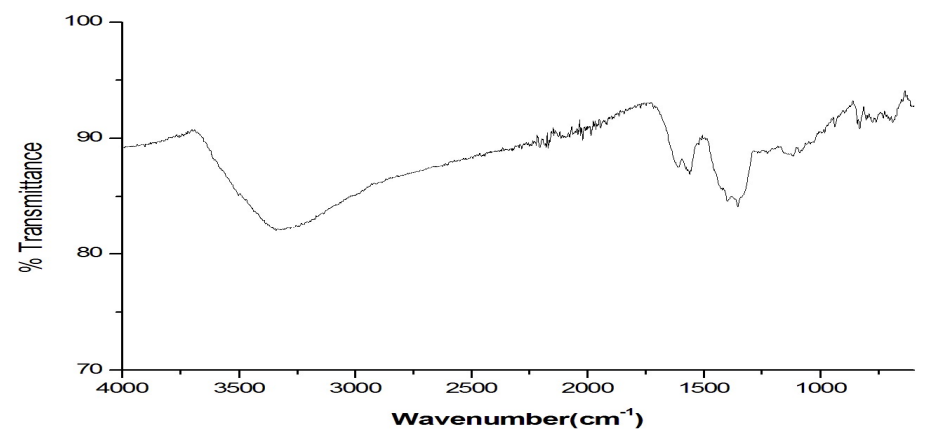

Fig. -4 


\section{Antibacterial Study}

The antimicrobial activity of leaf extract and ZnO NPs were investigated against Gram-negative (E.coli and P. mirabilis) and Gram-positive (S.epidermidis and B.thuringiensis ) bacteria by filter paper disc diffusion method and is shown in given Fig.-5 and 6. The biosynthesized ZnO NPs using Barrintonia Acutangula leaf extract exhibited vigorous antibacterial activity against both Gram-positive and Gramnegative bacteria strains. The previous research report also indicated that bactericidal activity of $\mathrm{ZnO}$ nanoparticles was more significant on Gram-positive than Gram-negative bacteria. ${ }^{21}$ The difference in sensitivity between Gram-positive and Gram-negative bacteria might be attributed to the differences in morphological constitutions between these microorganisms. The zone of inhibition of test samples was determined and compared with a control. The results revealed that ZnO NPs showed better activity against Gram+ve and Gram-ve bacteria. The Gram positive bacteria, S.epidermidis $(11 \mathrm{~nm})$ show more activity than B.thuringiensis $(7 \mathrm{~nm})$. P.mirabilis $(10 \mathrm{~nm})$ is more active than E.coli $(8 \mathrm{~nm})$ in the gram negative strains. The plant extract activity is low compared with the $\mathrm{ZnO}$ NPs synthesized from Barrintonia Acutangula extract.

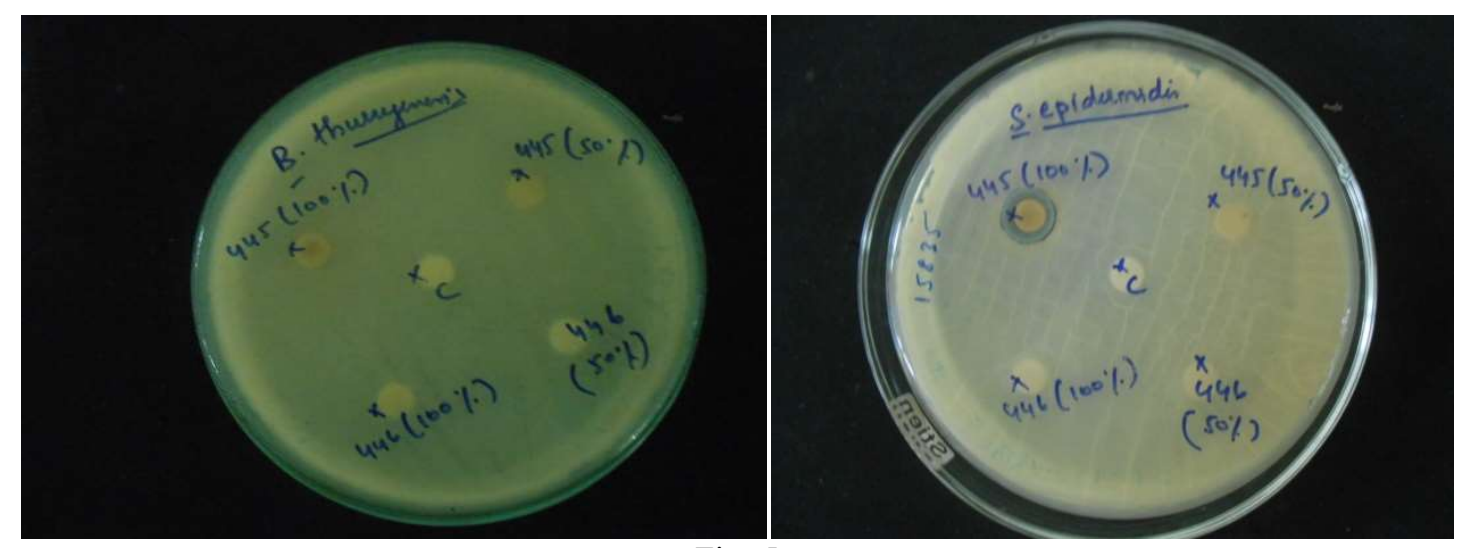

Fig.-5
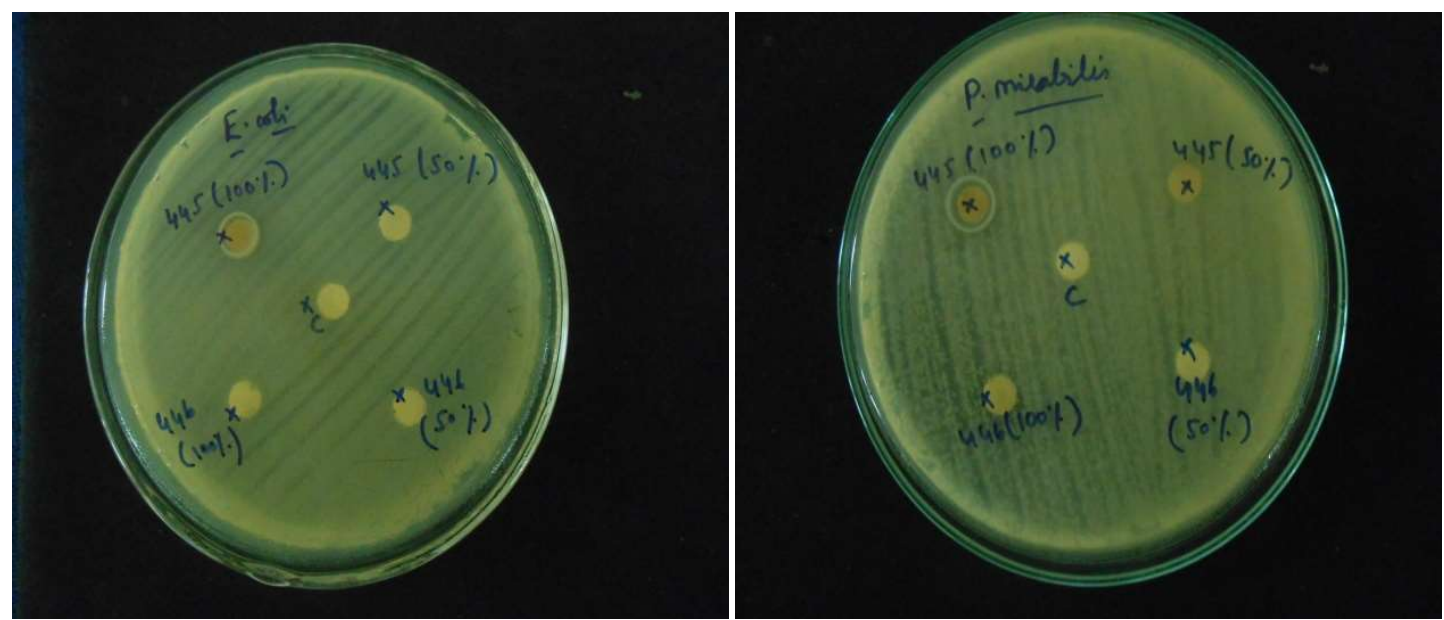

Fig.-6

\section{CONCLUSION}

In this study, the rapid biological synthesis of zinc oxide nanoparticles $(\mathrm{ZnO})$ was achieved by applying a novel and straightforward green chemistry procedure involving Barrintonia Acutangula leaf extract as a reducing and capping agent. The use of plant extracts avoids the usage of harmful and toxic reducing and stabilizing agents. The synthesized nanocrystallites of $\mathrm{ZnO}$ are in the range of 11-25 $\mathrm{nm}$ with a spherical shape. FTIR, XRD, S.E.M., and U.V. Visible analyses confirmed the successful formation of zinc oxide nanoparticles. The environmental conditions will affect the stability of nanoparticle and agglomerates are formed. The obtained nanoparticles are potential to be developed as an antibacterial agent. Further, the 
RASĀYAN J. Chem.

Vol. 14 | No. 3 |1653-1658| July - September | 2021

biosynthesized $\mathrm{ZnO}$ nanoparticles using Barrintonia Acutangula leaf extract proved to be effective antibacterial agents against both Gram-positive (S.epidermidis and B.thuringiensis and Gram-negative (E.coli and P.mirabilis) bacteria suggesting promising and robust action of green-synthesized $\mathrm{ZnO}$ nanoparticles against biological systems.

\section{ACKNOWLEDGEMENT}

The authors thank N.S.S. College, Pandalam, for the support to perform the research. The authors were very grateful to Sophisticated Analytical Instruments Facility, Kochi, India, for the analyses.

\section{REFERENCES}

1. S. Gunalana, R. Sivaraja and V. Rajendranb, Progress in Natural Science: Materials, 22(6), 693 (2012), https://doi.org/10.1016/j.pnsc.2012.11.015

2. R. Damayanti, Tamrin, Z. Alfian and Eddiyanto, Rasayan Journal of Chemistry, 13(4), 2483 (2020), http://dx.doi.org/10.31788/RJC.2020.1345792

3. S. Tachikawa, A. Noguchi, T. Tsuge, M. Hara, O. Odawara and H. Wada, Materials, 4, 1132(2011), http://dx.doi.org/10.3390/ma4061132

4. P. Raveendran, J.Fu and S.L.Wallen, Journal of American Chemical Society, 125, 13940 (2003), http://dx.doi.org/10.1021/ja029267j

5. A. H. Wani and M. A. Shah, Journal of Applied Pharmaceutical Science, 2(3), 40(2012), http://dx.doi.org/10.1007/s11051-009-9835-3

6. A. Raj and R. Lawerence, Rasayan Journal of Chemistry, 11(3), 1339(2018), http://dx.doi.org/10.31788/RJC.2018.1132009

7. R. Yuvakkumar, J. Suresh, A. J. Nathanael, M. Sundrarajan and S.I. Hong, Materials Science and Engineering: C, 17, (2014), http://dx.doi.org/10.1016/j.msec.2014.04.025

8. C. Dagdeviren, S.W. Hwang, Y. Su, S. Kim, H. Cheng, O. Gur, R. Haney, F.G. Omenetto, Y. Huang and J. A. Rogers, Materials Science and Engineering,9(20), 3398,( 2013).

9. L. Wang, Y. Kang, X. Liu, S. Zhang, W. Huang and S. Wang, Sensors Actuators B, 162, 237, (2012), https://doi.org/10.1016/j.snb.2011.12.073

10. M. G. Demissie, F. K. Sabir, G. D. Edossa, and Bedasa, Journal of Chemistry, (2020) https://doi.org/10.1155/2020/7459042

11. S. E. Cross, B. Innes, M. S. Roberts, T. Tsuzuki, T. A. Robertson and P. McCormick, Skin Pharmacology and Physiology, 20, 148,( 2007), https://doi.org/10.1159/000098701

12. J. Zhou, N. S. Xu and Z. L. Wang, Advanced Materials, 18, 2432(2006), https://doi.org/10.1002/adma.200600200

13. S. Gunalan, R. Sivaraj and V. Rajendran , Natural Science: Materials International, 22, 693(2012), https://doi.org/10.1016/j.pnsc.2012.11.015

14. A. M. Getie, A. Belay, A. R. Chandra Reddy, and Z. Belay, Journal of Nanomedicine \& Nanotechnology, 8(9), 2157(2017), http://dx.doi.org/10.4172/2157-7439.S8-004

15. G. M. Srirangam and K. P. Rao, Rasayan Journal of Chemistry, 10(1), 46(2017), http://dx.doi.org/10.7324/RJC.2017.1011548

16. S. Vijayakumar, B. Vaseeharan, B. Malaikozhundan, and M. Shobiya, Biomedicine \& Pharmacotherapy, 84, 1213(2016), https://doi.org/10.1016/j.biopha.2016.10.038

17. P. M. Pranjali, M. P. Pooja and J. D. Maruti, Biochemistry and Biophysics Reports, 17, 71(2019).

18. S. Elias, G. Elham, G. and N. Kazem, International Journal of Molecular Science, 14(4), 7880(2013).

19. R. Yuvakkumar, J. Suresh, B. Saravanakumar, A. J. Nathanaeld, S. I. Honga, and Rajendran, Spectrochimica Acta Part A: Molecular and Biomolecular Spectroscopy, 137, 250(2015).

20. M. Awwad, B. Albiss, and L. Ahmad, Advanced Materials Letters, 5, 520(2014), http://dx.doi.org/10.5185/amlett.2014.590

21. A. Tayel, W. El-Tras, S. Moussa, A. El-Baz, and H. Mahrous, Journal of Food Safety, 31(2), 211(2011), https://doi.org/10.1111/j.1745-4565.2010.00287.x

[RJC-6332/2020] 